\title{
Low Threshold Quantum Dot Lasers
}

\author{
Veena Hariharan Iyer, Rekha Mahadevu and Anshu Pandey*
}

Solid State and Structural Chemistry Unit, Indian Institute of Science, Bangalore 560012, India.

\section{MATERIALS AND METHODS}

Synthetic methods. CHEMICALS: Tellurium (Te, 99.99\%), zinc acetate dihydrate $\left(\mathrm{Zn}(\mathrm{ac})_{2} .2 \mathrm{H}_{2} \mathrm{O}, 99.0 \%\right)$, sodium borohydride $\left(\mathrm{NaBH}_{4} 96 \%\right)$, trioctylphosphine (TOP, technical grade, 90\%), 1-octadecene (ODE, technical grade, 90\%), oleylamine (technical grade, 70\%), oleic acid (technical grade, 90\%), sulphur (S, <99.5\%), zinc stearate (purum, 10-12\% zinc basis), selenium (Se, 99.99\%), trioctylphosphine oxide (TOPO, technical grade, 90\%), tetradecylphosphonic acid (TDPA, 97\%), 1-dodecanethiol (DDT, >98\%) and cadmium oxide (99.9\%) were purchased from Sigma-Aldrich. 1,4-Butanediol (90\%, AR) and dextrose (95\% anhydrous, AR) were purchased from SD Fine Chemicals. All chemicals were used without further purification.

\section{PREPARATION OF PRECURSORS:}

(a) $0.1 \mathrm{M}$ cadmium oleate: $0.3210 \mathrm{~g}$ of cadmium oxide, $3 \mathrm{ml}$ of oleic acid and $3 \mathrm{ml}$ of ODE are added to a flask. The contents are held under vacuum at $100{ }^{\mathrm{O}} \mathrm{C}$ for 1 minute and then the flask is filled with argon. Temperature is raised to $250{ }^{\circ} \mathrm{C}$ for 10 minutes to convert cadmium oxide to cadmium oleate. Mixture is cooled and $18 \mathrm{ml}$ ODE and $1 \mathrm{ml}$ of oleylamine are injected to produce $0.1 \mathrm{M}$ cadmium oleate solution. 
(b) $0.1 \mathrm{M}$ zinc-oleate solution: $548.78 \mathrm{mg}$ of zinc acetate dihydrate, $5 \mathrm{ml}$ of oleic acid and 3 $\mathrm{ml}$ of ODE are added to a flask. The contents are held under vacuum at $100{ }^{\circ} \mathrm{C}$ for 1 minute and the flask is filled with argon. Temperature is raised to $250{ }^{\circ} \mathrm{C}$ for 10 minutes to convert zinc acetate to zinc oleate. Mixture is cooled and $16 \mathrm{ml} \mathrm{ODE}$ and $1 \mathrm{ml}$ of oleylamine are injected to produce $0.1 \mathrm{M}$ zinc oleate solution

(c) $0.1 \mathrm{M} \mathrm{S}$ in ODE: $64 \mathrm{mg}$ of elemental $\mathrm{S}$ is added to $20 \mathrm{~mL}$ of ODE and stirred overnight at room temperature to produce $0.1 \mathrm{M}$ solution of $\mathrm{S}$.

(d) $\underline{0.25 \mathrm{M} \text { Te in TOP: }} 0.3166 \mathrm{~g}$ of Te pieces and $10 \mathrm{ml}$ of TOP is stirred for 12 hours to produce a $0.25 \mathrm{M}$ solution of Te.

(e) Precursors for Zinc selenide growth: $1264.7 \mathrm{mg}$ of zinc stearate, $156 \mathrm{mg}$ of Se and $8 \mathrm{~mL}$ ODE is added to a flask and stirred for 10 hours to make $0.25 \mathrm{M}$ of zinc and Se precursors in the form of a slurry.

(f) TOPO/ TDPA in ODE: $0.1 \mathrm{~g}$ of TDPA and $0.3 \mathrm{~g}$ of TOPO is dissolved in $10 \mathrm{ml}$ of ODE by stirring overnight.

The heterostructure nanocrystal is prepared under argon atmosphere. Oleylamine is used as ligand and ODE is used as the solvent medium. The preparation of $\mathrm{ZnTeSe} / \mathrm{ZnSe} / \mathrm{CdZnS}$ is carried out in two steps. The first step involves the formation of ZnTeSe or ZnTe core and the second step involves formation of intermediate and outer shell.

\section{Preparation of ZnTeSe core:}

$94.5 \mathrm{mg}$ of sodium borohydride and $7.8 \mathrm{mg}$ of Se is transferred to a flask filled with argon. $1.6 \mathrm{ml}$ of Te in TOP prepared as above is injected into the flask along with $100 \mu \mathrm{l}$ of 1,4- 
butanediol and the temperature of the reaction mixture is raised to $75^{\circ} \mathrm{C}$ for $5 \mathrm{~min}$. The flask is then cooled to room temperature and $90 \mathrm{mg}$ of dextrose dissolved in $1 \mathrm{~mL}$ of 1,4butanediol is added in order to consume the unreacted borohydride. Any unreacted borohydride left in the flask decomposes at higher temperatures with the evolution of hydrogen gas.

$109.8 \mathrm{mg}$ of zinc acetate dihydrate, $8 \mathrm{ml}$ of ODE and $2 \mathrm{ml}$ of oleylamine is taken in the second flask. Reaction mixture is held under vacuum at $100^{\circ} \mathrm{C}$ and the flask is filled with argon. $40 \%$ of the contents of the first flask (Se-Te precursor prepared as mentioned above) is rapidly injected into the second flask containing zinc acetate. $60 \%$ of the remnant of flask 1 is then transferred to the second flask drop wise, while ensuring that the temperature of the mixture is at $100^{\circ} \mathrm{C}$.

\section{Preparation of ZnTe core:}

$94.5 \mathrm{mg}$ of sodium borohydride is transferred to a flask filled with argon. $2 \mathrm{ml}$ of $\mathrm{Te}$ in TOP prepared as above is injected into the flask along with $100 \mu \mathrm{l}$ of 1,4-butanediol and the temperature of the reaction mixture is raised to $75{ }^{\mathrm{O}} \mathrm{C}$ for $5 \mathrm{~min}$. The flask is then cooled to room temperature and $90 \mathrm{mg}$ of dextrose dissolved in $1 \mathrm{~mL}$ of 1,4-butanediol is added in order to consume the unreacted borohydride. Any unreacted borohydride left in the flask decomposes at higher temperatures with the evolution of hydrogen gas.

$109.8 \mathrm{mg}$ of zinc acetate dihydrate, $8 \mathrm{ml}$ of ODE and $2 \mathrm{ml}$ of oleylamine is taken in a second flask. Reaction mixture is held under vacuum at $100{ }^{\circ} \mathrm{C}$ and the flask is filled with argon. $40 \%$ of the content in the first flask (Te precursor prepared as mentioned above) is rapidly injected into the second flask containing zinc acetate. $60 \%$ of the remnant of flask 1 is then transferred to the second flask drop wise, while ensuring that the temperature of the mixture is at $100{ }^{\circ} \mathrm{C}$. 
Preparation of $\mathrm{ZnSe} / \mathrm{ZnCdS}$ shells on $\mathrm{ZnTe}$ and $\mathrm{ZnTeSe}$ cores:

$\mathrm{ZnTeSe}$ or ZnTe cores are prepared as mentioned above. The temperature of the reaction mixture is raised to $160{ }^{\circ} \mathrm{C}$ and $0.5 \mathrm{~mL}$ of zinc and Se precursor slurry prepared as aforementioned is added. Mixture is then annealed at $280^{\circ} \mathrm{C}$ for ten minutes. The temperature of the reaction mixture is then lowered to $160^{\circ} \mathrm{C}$ and $0.5 \mathrm{~mL}$ of zinc and Se precursor slurry is further added and mixture is again annealed at $280^{\circ} \mathrm{C}$ for ten minutes. This procedure is repeated until $\mathrm{ZnSe}$ shell of desired thickness is obtained (3 to 4 monolayers). $100 \mu \mathrm{l}$ of TOP is added to the reaction mixture to dissolve excess Se. The reaction mixture is then cooled to room temperature and the quantum dots are cleaned using methanol alone. Cleaned dots are then dispersed in minimum amount of hexane and transferred to a flask. $1 \mathrm{~mL}$ oleylamine and $4 \mathrm{~mL}$ ODE are added to the flask and the mixture is held under vacuum for 100 seconds at $100{ }^{\mathrm{O}} \mathrm{C}$ and is then filled with argon. A 1:1 solution of $0.1 \mathrm{M}$ cadmium oleate and $0.1 \mathrm{M} \mathrm{Zn-}$ oleate is prepared by mixing two cation precursors. $1 \mathrm{~mL}$ of $0.1 \mathrm{M}$ cation precursor and $1 \mathrm{ml}$ of $0.1 \mathrm{M} \mathrm{S}$ in $\mathrm{ODE}$ is added drop wise to the reaction mixture at $240{ }^{\circ} \mathrm{C}$ in presence of $1 \mathrm{~mL}$ of DDT. This is followed by addition of $0.5 \mathrm{~mL}$ of $0.1 \mathrm{M}$ cadmium oleate and $0.5 \mathrm{M} \mathrm{S}$ in ODE. $1 \mathrm{~mL}$ of TOPO/TDPA in ODE, $100 \mu \mathrm{l}$ of TOP and $0.5 \mathrm{~mL}$ cadmium oleate is then added. Drop wise addition of cadmium oleate and S in ODE is continued until PL maxima reaches $800 \mathrm{~nm}$.

Quantum dots thus obtained were characterized using powder XRD, TEM, and optical techniques.

Characterization of CdZnS layer: Powder x-ray diffraction was used to understand the effect of DDT in the preparation of $\mathrm{ZnCdS}$ alloy layer. A $\mathrm{ZnSe}$ core was prepared. To prepare ZnSe QDs, $13.2 \mathrm{mg}$ of zinc acetate dihydrate, $25.3 \mathrm{mg}$ of zinc stearate and $23.7 \mathrm{mg}$ of selenium were transferred to a flask containing $4 \mathrm{~mL}$ of ODE and $2 \mathrm{~mL}$ of oleylamine. The 
reaction mixture was held under vacuum at $100{ }^{\circ} \mathrm{C}$ for 2 minutes and then filled with argon. The temperature of the reaction mixture was raised to $240{ }^{\circ} \mathrm{C}$ and annealed for 5 minutes. 100 $\mu l$ of TOP was added and the reaction mixture was cooled. The ZnSe QDs thus prepared were cleaned using methanol and acetone. Finally, absorption spectrum of cleaned $\mathrm{ZnSe}$ core was recorded.

The cleaned ZnSe QDs were then split into two portions. The first portion was dissolved in $4 \mathrm{~mL}$ ODE and $2 \mathrm{~mL}$ oleylamine and transferred to a flask. The flask was held under vacuum at $100{ }^{0} \mathrm{C}$ for 2 minutes and then filled with argon. The temperature of the reaction mixture was raised to $240{ }^{0} \mathrm{C} .3 \mathrm{~mL}$ of $1: 3,0.1 \mathrm{M}$ solution of Cadmium oleate and zinc oleate was added along with $3 \mathrm{~mL} 0.1 \mathrm{M} \mathrm{S}$ in ODE in a drop wise manner. The flask was cooled and the QDs thus obtained were cleaned using methanol and ethanol.

The second portion of parent cleaned ZnSe QDs was dissolved in $4 \mathrm{~mL}$ ODE and $2 \mathrm{~mL}$ oleylamine and transferred to a flask. The flask was held under vacuum at $100^{\circ} \mathrm{C}$ for 2 minutes and then filled with argon. The temperature of the reaction mixture was raised to $240^{\circ}$ C. $0.25 \mathrm{~mL}$ of DDT was injected and $1 \mathrm{~mL}$ of $1: 3,0.1 \mathrm{M}$ solution of Cadmium oleate and zinc oleate was added along with $1 \mathrm{~mL} 0.1 \mathrm{M} \mathrm{S}$ in ODE in a drop wise manner. Another $0.25 \mathrm{~mL}$ of DDT was injected followed by drop wise addition of $1 \mathrm{~mL}$ of zinc, cadmium (3:1) and sulphur precursors. This was repeated one more time and the heating was discontinued. The flask was cooled and the QDs thus obtained were cleaned.

A uniform film of quantum dots prepared in the presence and in the absence of DDT was deposited on a glass substrate and powder XRD pattern was recorded. The XRD pattern suggests that in the absence of DDT, the peak position of QDs overlap with CdS. In presence of DDT, the position of peak of CdZnS QDs lies in between CdS and $\mathrm{ZnS}$. 
Power Dependence of QD Emission spectrum: ZnTe/ZnSe/ZnCdS QDs were dispersed in hexane and were illuminated with $405 \mathrm{~nm}$ light of different power. The intensity of the peak at lower energy is dependent on the illumination power and increases as power of the source increases.

\section{Determination of absorption cross-sections and lifetime:}

Quantum dots were cleaned and dissolved in hexane to obtain absorption spectra. The solvent was then evaporated, and the NCs were digested in aqua regia (solution of concentrated $\mathrm{HCl}$ and $\mathrm{HNO}_{3}$ such that $\mathrm{HCl}: \mathrm{HNO}_{3}=1: 3$ ). The resultant solution was diluted to $25 \mathrm{~mL}$. Composition was then determined using inductively coupled plasma. Size distributions were estimated from TEM images. The density of NCs was calculated by multiplying the mole fraction of each semiconductor layer by their corresponding density. Quantum dots used in this work are typically less than $15 \mathrm{~nm}$ in size. Semiconductor particles of this size do not scatter perceptibly. Extinction and absorption have thus been taken to be equal throughout this work. The cross-section of the quantum dots used in figures 3 and 4 in the main manuscript at $400 \mathrm{~nm}$ is determined to be $8.8 \times 10^{-14} \mathrm{~cm}^{2}$. Using the measured cross-sections and lifetimes, we estimate 0.02 excitons per quantum dot at threshold in figure $3 \mathrm{~b}$ of the main manuscript. Lifetime has been extracted by fitting the decay curve to

exponentials, and calculating an average lifetime through $\langle\tau\rangle=\frac{\sum a_{i} \tau_{i}^{2}}{\sum a_{i} \tau_{i}}$, where $a_{i}$ is the amplitude of an exponential and $\tau_{\mathrm{i}}$ is the associated lifetime. 


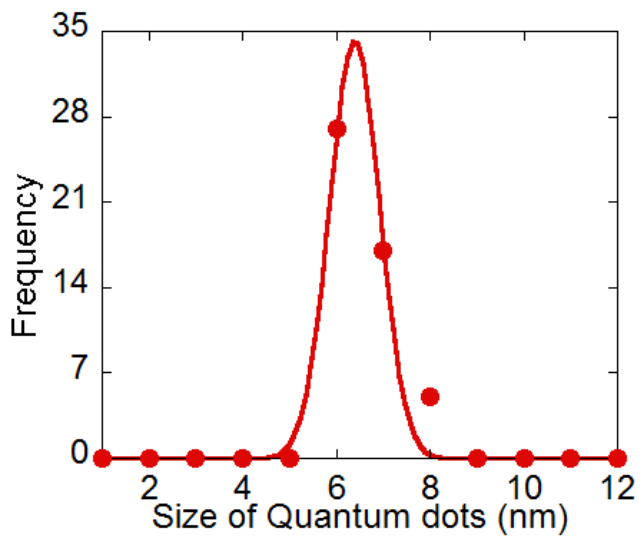

Figure S1. Histogram of size distribution of quantum dots used for figures $2 \mathrm{c}-\mathrm{d}$ in the main text.

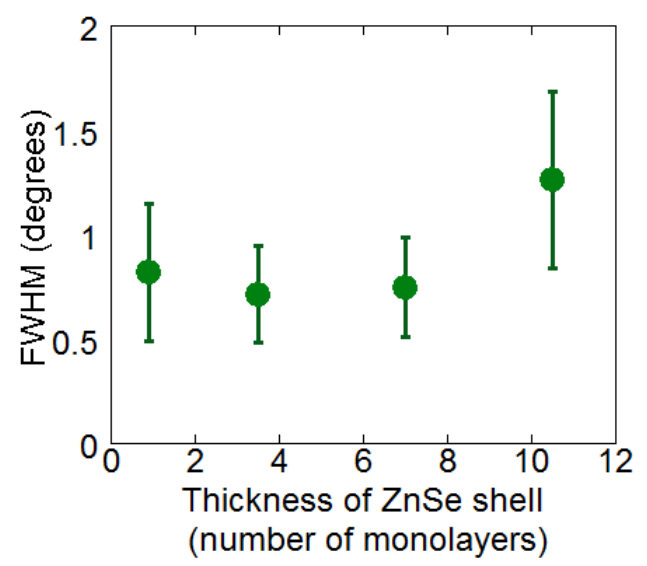

Figure S2. The linewidth of the ZnTe core patterns are observed to be constant during the outer ZnSe shell growth. This implies that within the errors of this measurement, the core sizes are unchanged during ZnSe shell growth, and therefore, ZnSeTe interface alloys are absent to within our detection limits. This data corresponds to the $\left(2 \theta=50^{\circ}\right)$ peak in figure $1 \mathrm{c}$ of the main manuscript. 

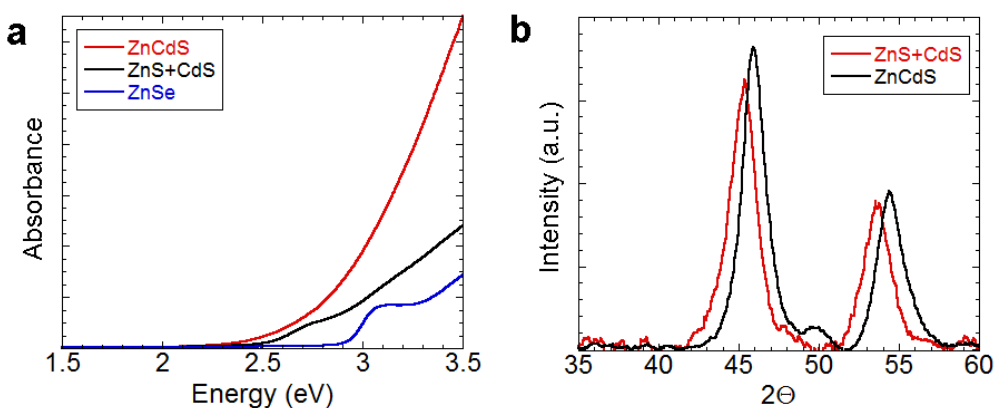

Figure S3: (a)Absorbance of ZnSe core (blue), ZnS and CdS shell synthesized in absence of DDT (black) and ZnCdS alloy synthesized in presence of DDT (red) (b) Powder XRD pattern (in degrees) of ZnSe core coated with $\mathrm{ZnS}$ and CdS shell in presence (black) and absence (red) of DDT.

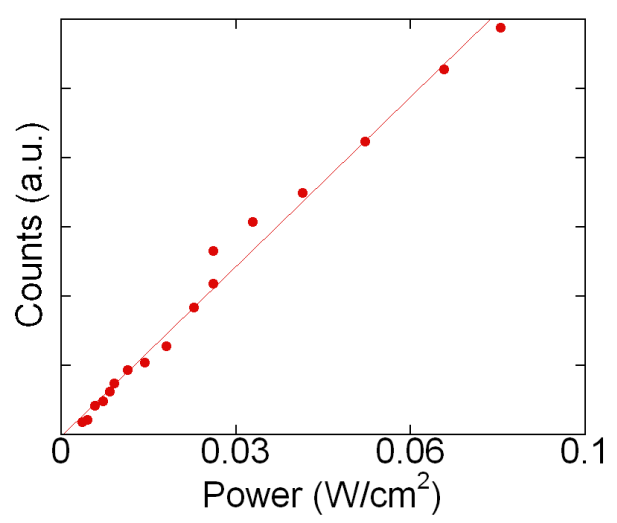

Figure S4. Performance of the setup used to generate figure $2 \mathrm{~d}$ in the main text. The data points represent the collected scatter from a reflective screen. The line makes an X-axis intercept of $8 \times 10^{-2} \mathrm{~mW} / \mathrm{cm}^{2}$ and thus provides us with an estimate of the least count of this setup. Note that our measured threshold in figure $2 \mathrm{~d}$ is 11 $\mathrm{mW} / \mathrm{cm}^{2}$ that is more than two orders of magnitude higher than the least counts of the setup. 


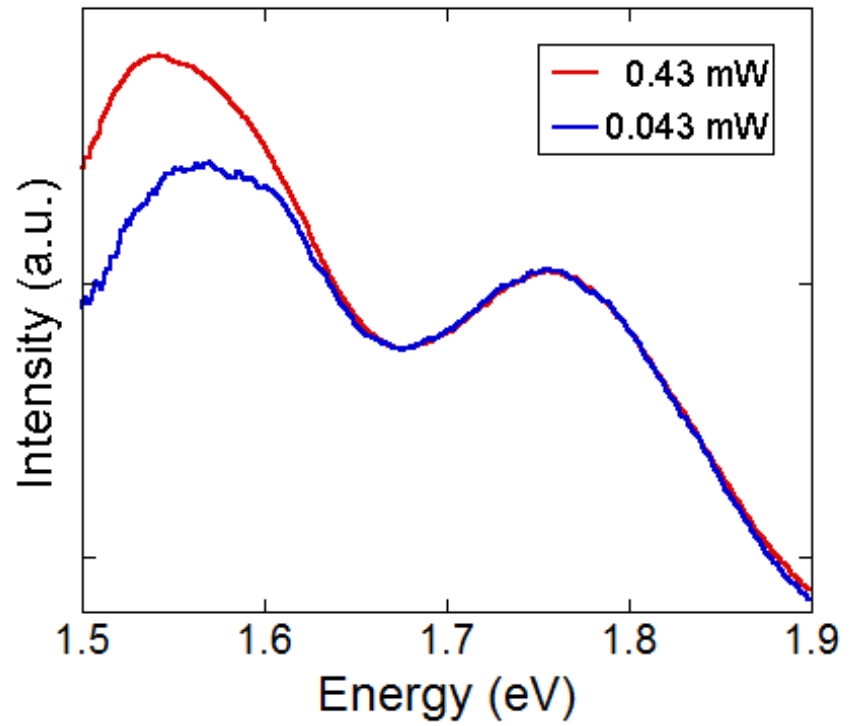

Figure S5: Normalized emission spectra (at $1.78 \mathrm{eV}$ ) of $\mathrm{ZnTe} / \mathrm{ZnSe} / \mathrm{ZnCdS}$ quantum dot at different powers of $405 \mathrm{~nm}$ excitation derived from a $450 \mathrm{~W}$ Xenon Lamp. 
a

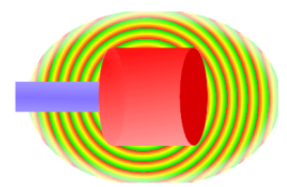

Spontaneous Emission/ Amplified Spontaneous Emission

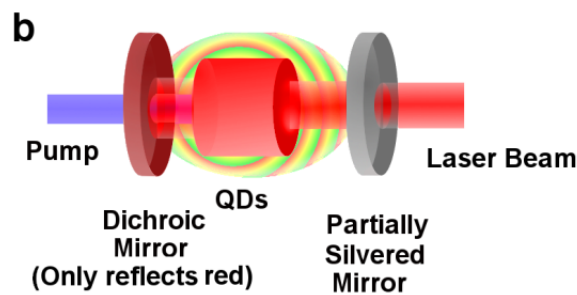

C

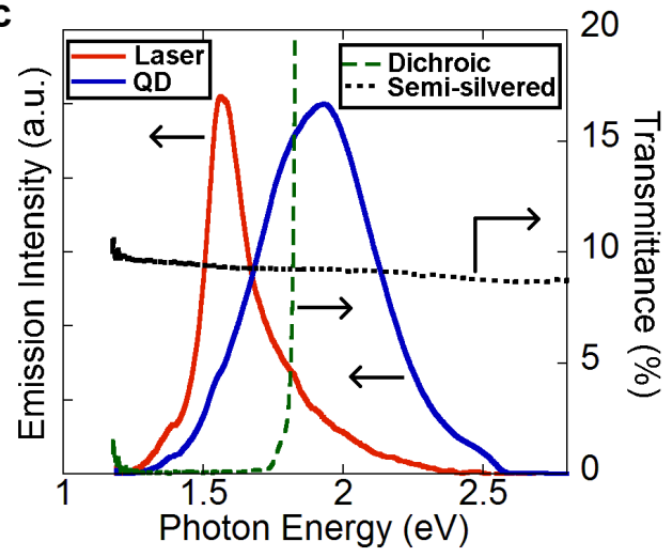

Figure S6 a. Pumping a bare QD film may lead to spontaneous emission or amplified spontaneous emission depending on the pump rate. b. Surrounding the same film with a pair of mirrors changes the emission characteristics depending on the reflection characteristics of the mirrors. In our case, we employ a mirror that reflects the redder components of light (dichroic), as well as a partially silvered mirror. The pump (405 nm) enters through the dichroic. The sample of QDs initially emits light as in case a. However, the dichroic causes the selective buildup of redder components of light within the resonator. At equilibrium above threshold, stimulated emission dominates, giving rise to a laser beam. The color of the beam is given by the colors of light at which the resonator is least lossy as well as the color of emission of the sample. The resonator thus picks up and amplifies a small sub-section of colors of light that the sample is capable of emitting. c. Actual emission data. The spectrum of the laser beam is given by the red curve. The emission of a QD film in absence of the resonator is given by the blue curve. Note that the laser beam still corresponds to the amplification of a selection of colors over which the cavity is the least lossy and the sample is emissive. Thus, the laser emission shows a strong cutoff to the lower energy side since the sample emission falls to zero. On the higher energy side, the laser emission drops to zero because the dichroic becomes increasingly lossy. Note that the laser emission has been collected from the silvered mirror side, and thus is an accurate description of the electromagnetic radiation present within the cavity. There are no other wavelength selecting features such as Fabry-Perot modes in this particular device due to the larger mirror separation.

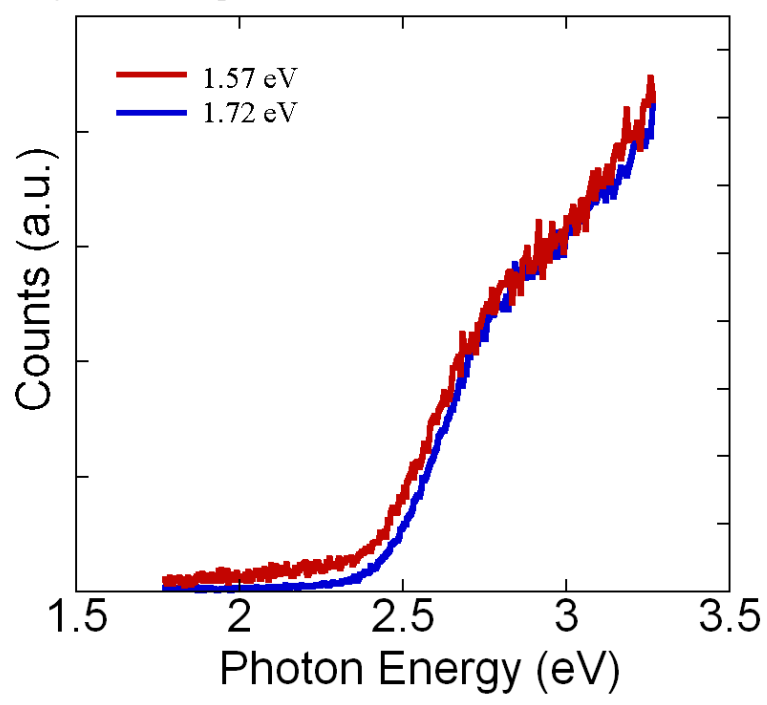

Figure S7. PLE of the sample in figure S6c at two different energy positions, exemplifying the sample homogeneity. 


\section{Power dependent spectra}

For purposes of sampling spontaneous as well as stimulated emission from a laser device, we employed a lossier cavity design. This involves the addition of an absorber layer between a QD film and the dichroic $800 \mathrm{~nm}$ mirror. Once again due to the greater separation of the two end mirrors, no Fabry-Perot modes are observed.
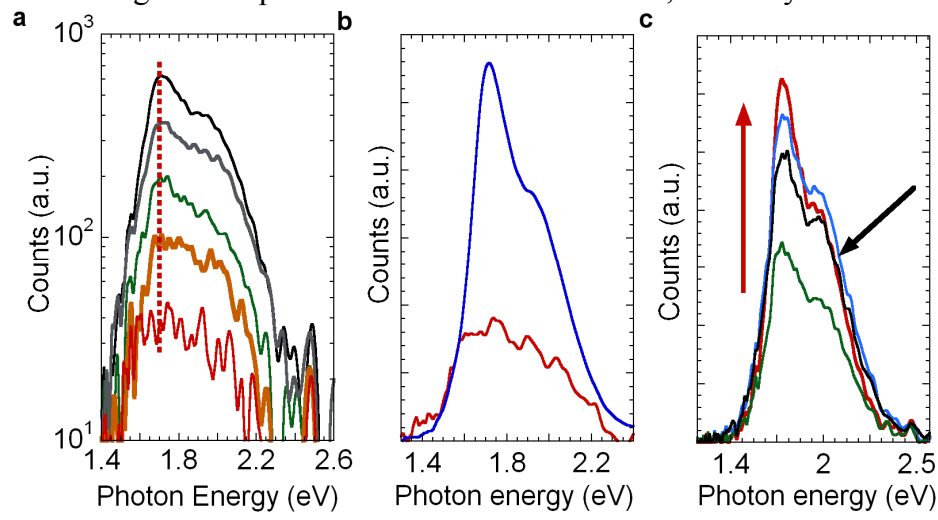

Figure S8. a. At increasing powers a sharp feature is observed in the emission spectrum with a threshold pump power of $0.32 \mathrm{~mW}$. b. A comparison of the emission below and above threshold. c. Following emergence of this feature, the normal spontaneous emission (black arrow) is clamped. Increasing power causes the sharp lasing feature to gain in amplitude. The red arrow corresponds to increasing pump power from a CW $405 \mathrm{~nm}$ diode.

\section{Kinetics from structured emission}
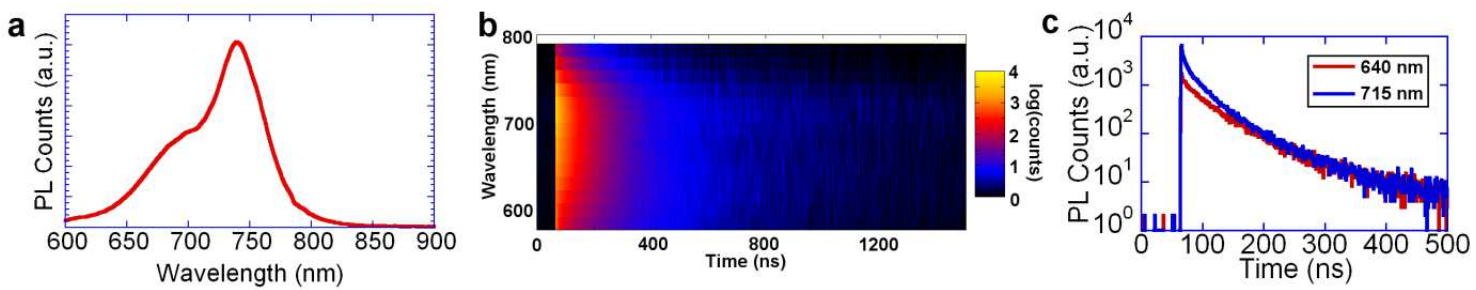

Figure S9 a. Structured emission from a QD sample. b. Decay kinetics as a function of wavelength. c. The sharper emission is seen to be associated with a faster kinetics. 


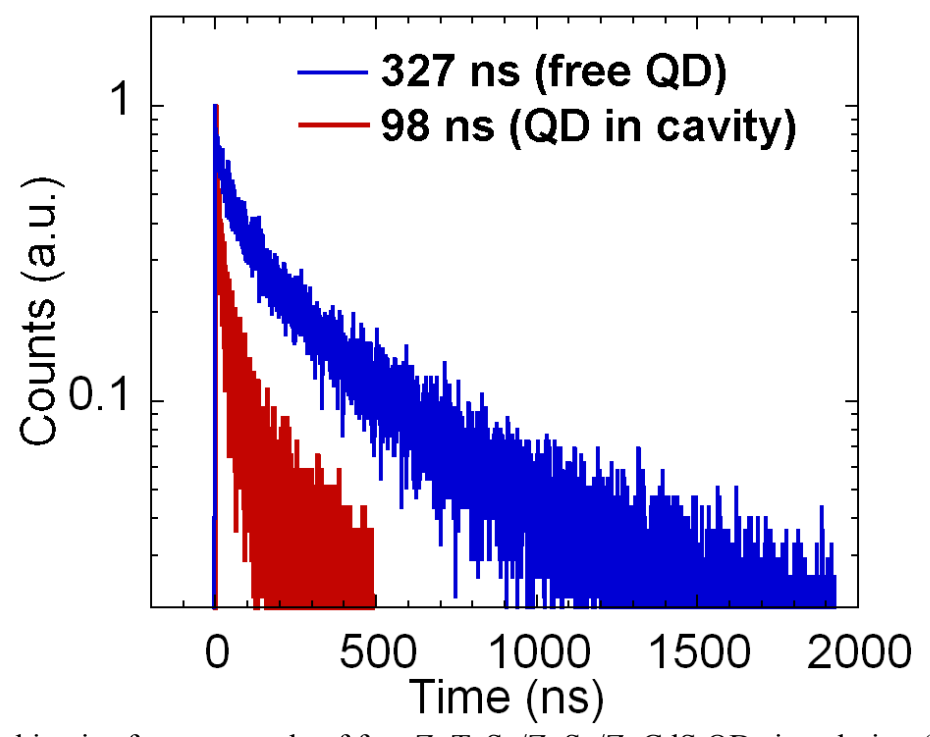

Figure S10. Emission kinetics from a sample of free ZnTeSe/ZnSe/ZnCdS QDs in solution (327 ns, blue) and in a cavity composed of a dichroic and a partially silvered ( $90 \%)$ mirror (98 ns, red).

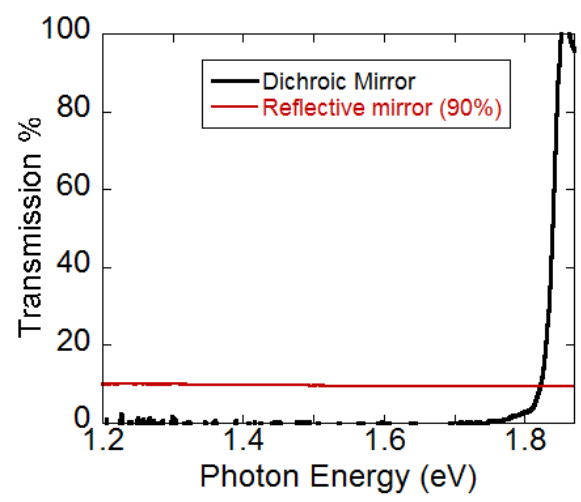

Figure S11: Transmission of Dichroic mirror (black) and partially reflecting silver mirror, 90\% (red). 


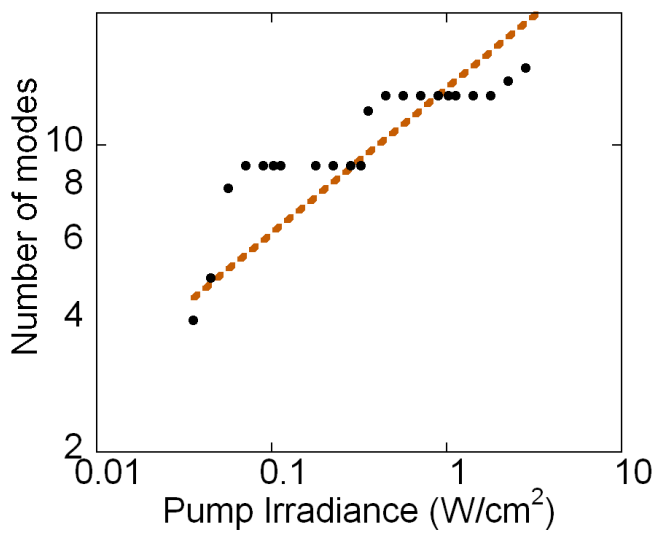

Figure S12. Number of modes observed in the multimode laser as a function of pump irradiance (black dots). The dashed line represents the expected number of modes in a multimode laser with an inhomogeneous gain medium based on the theory in reference 20 in the main text.

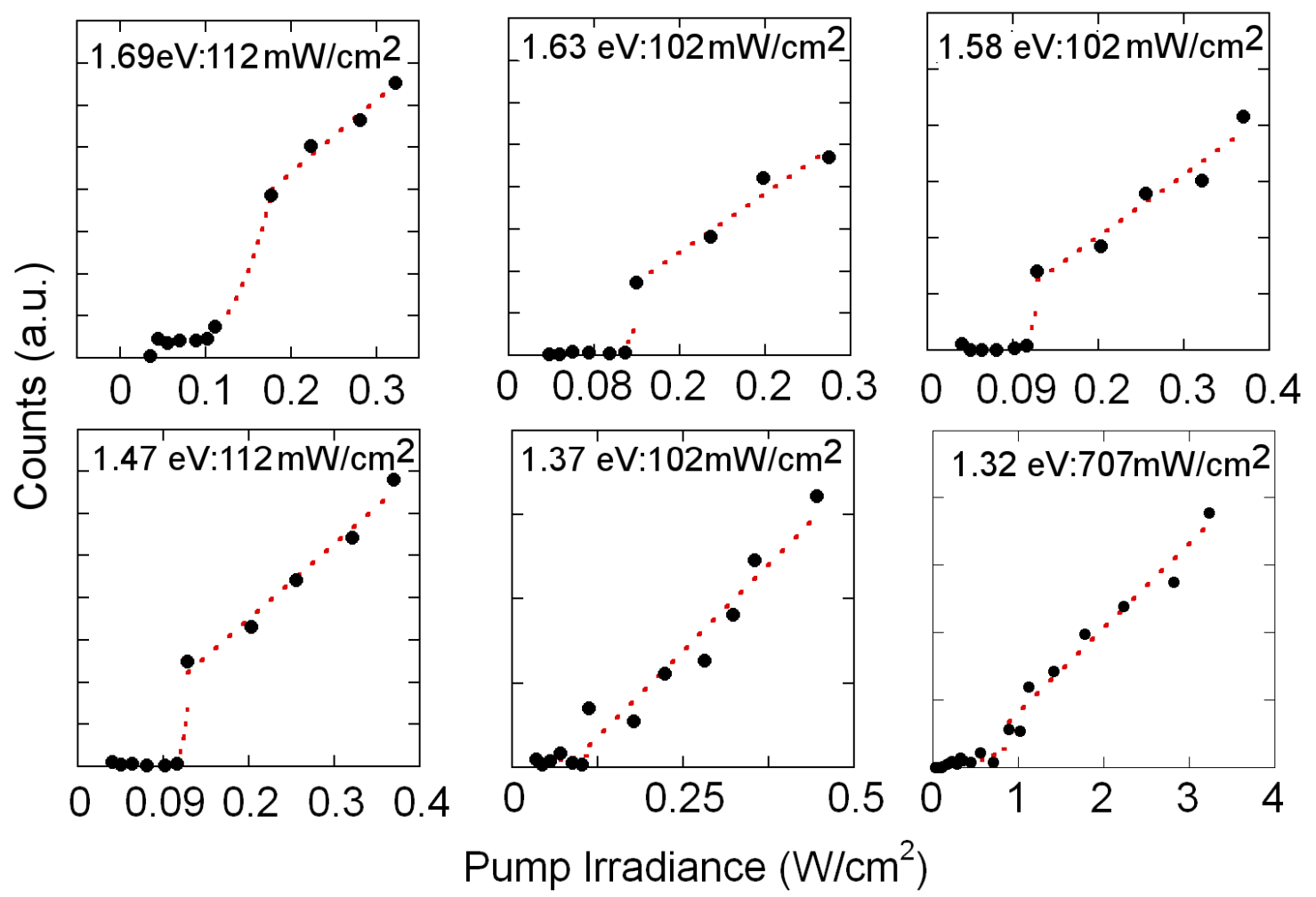

Figure S13. Thresholds of various modes in Figure 3a. The data have been fit to a multimode theory. Thresholds and energies of the modes are shown in each panel. These data have been extracted by fitting the output spectra to a Gaussian lineshape with a background. 


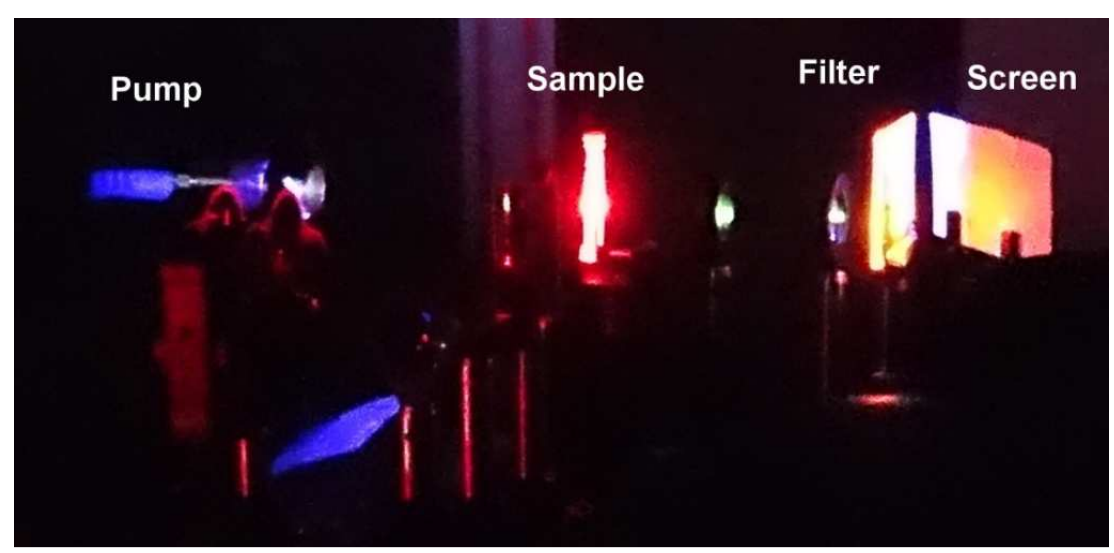

Figure S14. Photograph of an 87\% QY sample of ordinary QDs incapable of lasing. Notice the presence of strong, incoherent spontaneous emission from the sample on the screen. The pump delivers $90 \mathrm{~mW}$ to the sample at $405 \mathrm{~nm}(\mathrm{CW})$. The collection setup consists of two lenses, a long-pass filter and a screen.

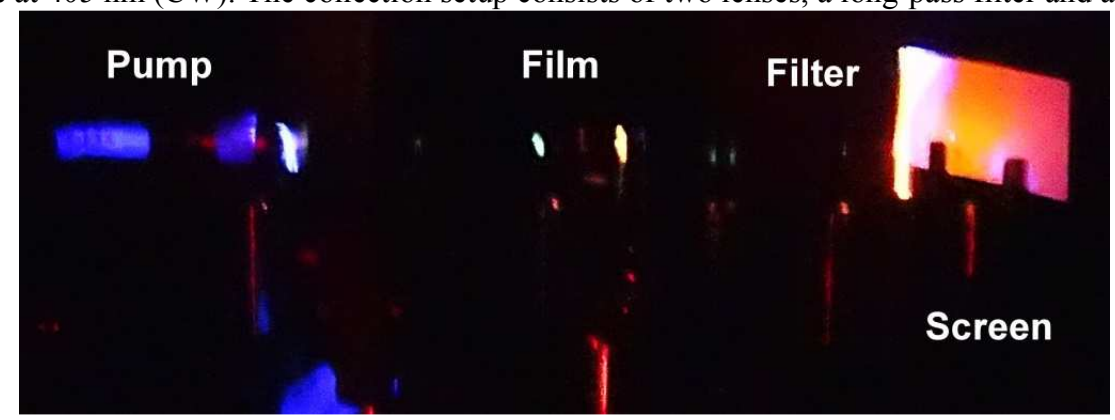

Figure S15. Photograph of a film of Lasing QDs (ZnTeSe/ZnSe/CdZnS), without a resonator. Notice that the emission is entirely directionless. The pump delivers $90 \mathrm{~mW}$ to the sample at $405 \mathrm{~nm}$. The film has a quantum yield of $4 \%$. The collection setup consists of two lenses, a long-pass filter and a screen.

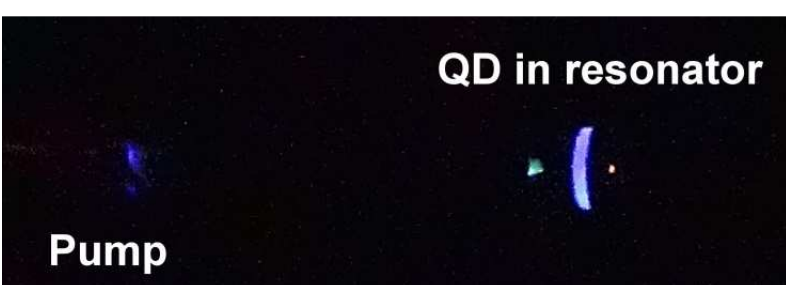

\section{Filter Screen}

Figure S16. Photograph of a film of Lasing QDs (ZnTeSe/ZnSe/CdZnS, same as above figure) inside a resonator, below lasing threshold. The emission is directionless and weak. The pump delivers $1 \mathrm{~mW}$ to the sample at $405 \mathrm{~nm}$. The resonator is composed of an $800 \mathrm{~nm}$ dielectric mirror and a $90 \%$ reflective metallic mirror. The collection setup consists of two lenses, a long-pass filter and a screen. 


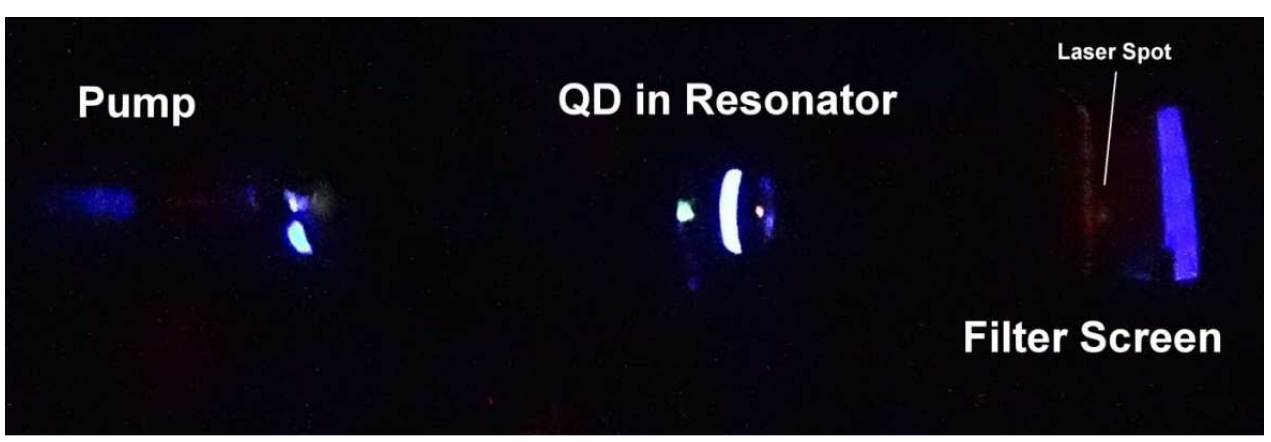

Figure S17. Photograph of a film of Lasing QDs(same as above) inside a resonator, just above lasing threshold. A faint but well defined red spot is observable corresponding to the laser beam from the QD laser. The pump delivers $25 \mathrm{~mW}$ to the sample at $405 \mathrm{~nm}$. The collection setup consists of two lenses, a long-pass filter and a screen.

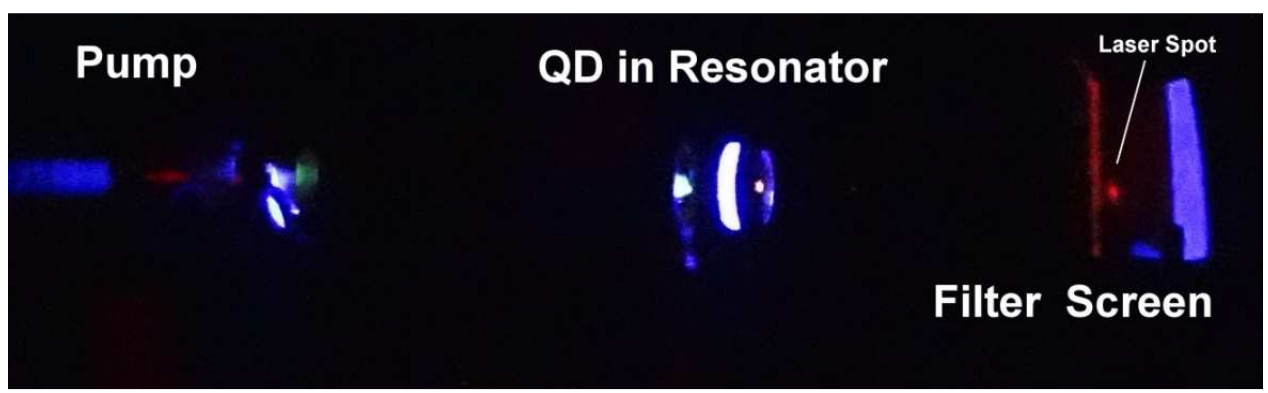

Figure S18. Photograph of a film of Lasing QDs inside a resonator, above lasing threshold. A bright red beam is observed. The pump delivers $46 \mathrm{~mW}$ to the sample at $405 \mathrm{~nm}$. The collection setup consists of two lenses, a long-pass filter and a screen.

\section{Quantum Yield Measurements:}

Quantum yields were determined through an absolute method, i.e. by employing an integrating sphere to make measurements. An Edinburgh Instruments FLS 920 Spectrometer with a working range of $\sim 300-1700 \mathrm{~nm}$ was used for these measurements. Reference spectra were first collected by putting solvent into the integrating sphere. The sample was subsequently introduced into the integrating sphere as a hexane dispersion. All samples investigated in this work exhibited quantum yields as low as $2 \%$ and as high as $10 \%$. Film quantum yields were determined in a similar manner. Film QYs were observed to be similar. For example, the QD film employed in the case of Figure S15 exhibited a quantum yield of 4\%. The film has an optical density of 0.65 at $400 \mathrm{~nm}$. 


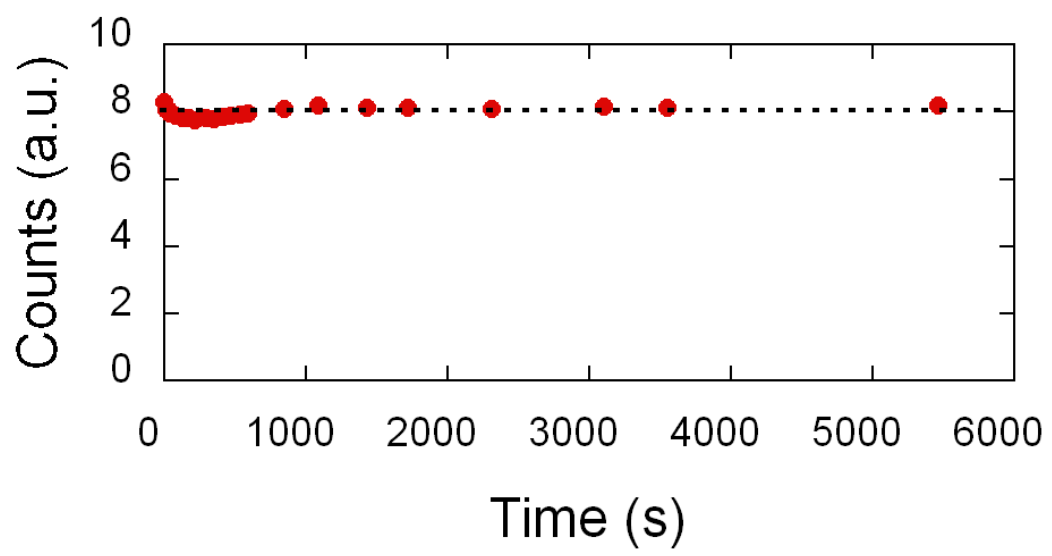

Figure S19. Plot of results of a device stability test. The QD Laser device has been illuminated by $586 \mathrm{~mW} / \mathrm{cm}^{2}$ at $405 \mathrm{~nm}$.

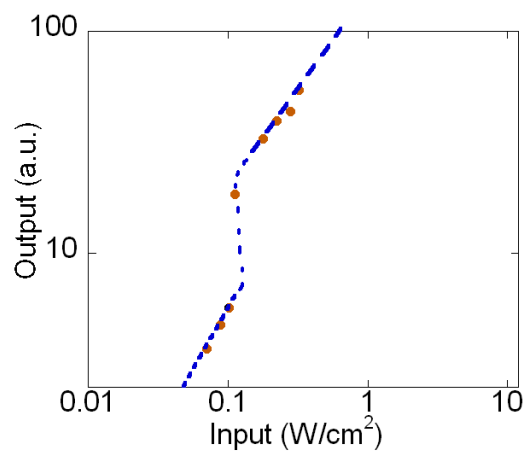

Figure S20. The laser input-output curve for the data shown in figure 3a. This corresponds to the $1.69 \mathrm{eV}$ mode with a threshold at $112 \mathrm{~mW} / \mathrm{cm}^{2}$. The dashed lines are guides to the eye. 